determinants. An idiotope present on the majority of immunoglobulins derived from the $\mathrm{V}_{\mathrm{H}} 4-34$ gene can be detected using the rat monoclonal antibody 9G4. 9G4 also allows the detection of B cells expressing $\mathrm{B}$ cell receptors derived from $\mathrm{V}_{\mathrm{H}} 4-34$ gene. We therefore determined the effect of $B$ cell depletion therapy with rituximab (RTX) on B cell subpopulations expressing the 9G4 idiotype in peripheral blood of patients with rheumatoid arthritis (RA) as a means of following the fate of a specific autoreactive $B$ cell population.

Materials and Methods B-cell subpopulations were characterised by flow cytometry using combinations of IgD, CD5, CD27 and CD38 in healthy controls (HC) $(n=7)$, patients with RA before $(n=10)$ and at clinical relapse after RTX $(n=17)$. The frequency of each 9G4+ B cell subpopulation was calculated after gating on 9G4+ cells.

Results No significant differences were observed in the frequency of total 9G4 + B cells between both patient groups and HC, although the levels of 9G4+ B cells tended to be higher at relapse after RTX treatment. After RTX treatment, repopulating total B cells were predominantly transitional ( $\operatorname{IgD}+\mathrm{CD} 38++$ ) and naïve $(\mathrm{IgD}+\mathrm{CD} 38+)$, with lower frequencies of most memory B-cell subpopulations. At relapse, RA patients had significantly more 9G4+IgD-CD38 - memory B cells compared to HC. This B cell subset was positively correlated with a 9G4+CD5+CD27+ subpopulation. The frequency of $9 \mathrm{G} 4+\mathrm{IgD}-\mathrm{CD} 38+$ memory $\mathrm{B}$ cells also tended to be increased after RTX compared to $\mathrm{HC}$ and preRTX. Lower levels of 9G4+IgD+CD38+ naïve, 9G4+IgD+CD38memory and 9G4+CD5-CD27+ memory B cells were found after RTX treatment compared with $\mathrm{HC}$ and pre-RTX. No significant differences were observed in $9 \mathrm{G} 4+\mathrm{IgD}+\mathrm{CD} 38++$ transitional, or 9G4+IgD-CD38++ plasmablasts in RA patients before and after RTX treatment in comparison with HC. However, there was a positive correlation between the frequency of 9G4+IgD-CD38++ plasmablasts and time after RTX treatment.

Conclusions Alterations in the frequency of memory B cell subpopulations expressing 9G4 idiotype occur in clinically relapsing RA patients after RTX treatment, particularly in IgD-CD38- memory $\mathrm{B}$ cell subset. It is possible that this $9 \mathrm{G} 4+\mathrm{B}$ cell subpopulation escapes B cell depletion therapy, being rescued in niches such as secondary lymphoid organs, or in inflammatory sites. Our results also suggest that the normal tolerance mechanisms preventing 9G4+ $B$ cells from becoming antibody producing cells is defective in patients with RA.

\section{A5.14 HOMOCITRULLINE-REACTIVE ANTIBODIES CAN BE GENERATED FROM SYNOVIAL B-CELLS FROM ACPA-NEGATIVE RA PATIENTS}

doi:10.1136/annrheumdis-2013-203219.14

'Fiona Murray, 'Lena Israelsson, 'Johanna Steen, 'Khaled Amara, 'Jing Shi, 'Lars Klareskog, ${ }^{2}$ Leendert Trouw, 'Vivianne Malmström. 'Rheumatology Unit, Department of Medicine at Karolinska University Hospital, Karolinska Institute, Solna, Stockholm, Sweden; ${ }^{2}$ Department of Rheumatology, Leiden University Medical Center, Leiden, The Netherlands

Background and Objectives Roughly two-thirds of rheumatoid arthritis (RA) patients carry antibodies, so-called ACPA, against peptides containing citrulline, a post-translationally modified version of the amino acid (AA) arginine in their sera and/or affected joints. A recent study reported the presence of antibodies against a different kind of post-translationally modified AA, homocitrulline, also known as carbamylated proteins (CarP), where lysine residues are altered by a non-enzymatic reaction involving cyanate. The authors reported IgG antibodies recognising homocitrullinated fibrinogen in the sera of $>45 \%$ of RA-patients and that $16 \%$ of ACPA-negative patients carried such anti-CarP antibodies.
Furthermore, in vivo studies describe that immunisation of mice with homocitrulline-containing peptides induced erosive arthritis. Thus, homocitrulline represents an interesting immune target in the context of RA. Therefore, we aimed to assess the proportion of anti-CarP antibodies sourced from the joints of RA patients with active disease.

Materials and Methods Using a single B cell-based cloning technology to isolate the immunoglobulin (Ig) genes from joint-derived $\mathrm{IgG}+\mathrm{CD} 19+\mathrm{B}$-cells, we then co-transfected the paired heavy and light chains into a human cell line and produced recombinant monoclonal antibodies from each individual B cell. This approach has previously allowed for the generation of citrulline-reactive monoclonal antibodies. Here, we analysed for reactivity to carbamylated (homocitrullinated) fibrinogen and compared to its counterpart unmodified fibrinogen, by ELISA. So far, we have analysed 42 monoclonal antibodies from three ACPA-positive patients and one ACPA-negative patient, for homocitrulline reactivity.

Results Between six and eleven antibodies were tested for homocitrulline activity for the ACPA-positive patients and eleven were examined for the ACPA-negative patient. All clones screened for CarP-reactivity were negative for citrulline binding. Antibodies were made up to concentrations of $5 \mathrm{ug} / \mathrm{ml}$ down to $0.63 \mathrm{ug} / \mathrm{ml}$ in a 4-step dilution. So far, one antibody displayed reactivity to the carbamylated peptide and this antibody originated from the ACPAnegative patient.

Conclusions Although the frequency of anti-CarP reactivity amongst the 42 antibodies examined does not match that previously reported for sera, the finding of one anti-CarP antibody from an ACPA negative patient, does support the earlier finding of significant frequencies of anti-Carp antibodies amongst ACPA-negative patients. It will be of great interest to expand the investigation for anti-Carp specificities, particularly among ACPA-negative patients and determine whether these antibodies could have pathological effects in RA patients.

\section{A5.15 HOW MICRORNAS CONTROL PLASMA CELLS}

doi:10.1136/annrheumdis-2013-203219.15

'Julia Schmid, 'Martina Porstner, 'Andreas Brandl, 'Edith Roth, ${ }^{2}$ Thomas Winkler, ${ }^{3}$ Michael Bösl, ${ }^{4}$ Andreas Radbruch, ' Jürgen Wittmann, 'Hans-Martin Jäck. 'Division of Molecular Immunology, Nikolaus-Fiebiger-centre, Department of Internal Medicine III, FAU Erlangen-Nürnberg, Erlangen, Germany; ${ }^{2}$ Section of Hematopoesis, Department of Biology, FAU Erlangen-Nürnberg, Erlangen, Germany; 'Max Planck Institute for Neurobiology, Martinsried, Germany; ${ }^{4}$ Deutsches Rheumaforschungszentrum (DRFZ), Berlin

Background and Objectives Our long-term goal is to understand how one class of interfering non-coding RNAs, the so-called microRNAs (miRNAs), regulates and fine-tunes the differentiation of mature B cells into effector cells, i.e., memory B cells and antibodysecreting plasma cells. miRNAs control the expression of specific target genes at the post-transcriptional level by binding to target sequences e.g., in the 3'-untranslated region of mRNAs, which, depending on the degree of the binding, results either in a block of translation or an accelerated degradation of the respective target mRNA. Lineage-specific deletion of the miRNA-processing DICER protein as well as of individual miRNAs revealed the importance of miRNA pathway in early steps of central B cell maturation. However, the mechanisms by which miRNA-dependent circuits control the antigen-induced phase of $B$ cell activation of mature naive $B$ cells and their subsequent differentiation into effector cells remain largely elusive.

Methods To change this situation we established a transgenic knock-in mouse line with a floxed allele of DGCR8, an essential subunit of the nuclear miRNA processing complex. B cell-specific deletion of DGCR8 resulted in a complete maturation block at the pro-B stage, indicating that miRNA processing is essential for central 